\title{
Errata
}

\section{Erratum: Tang et al., "Fast Vesicle Transport Is Required for the Slow Axonal Transport of Synapsin"}

In the article "Fast Vesicle Transport Is Required for the Slow Axonal Transport of Synapsin” by Yong Tang, David Scott, Utpal Das, Daniel Gitler, Archan Ganguly, and Subhojit Roy, which appeared on pages 15362-15375 of the September 25, 2013 issue, "PKA inhibitor H89" was incorrectly referred to as "PKA activator H89" twice: on page 15368, right column, first paragraph, line 19, in the sentence beginning, "In contrast, treatment of neurons with PKA activator H89 . .."; and in the last sentence of the Figure 6 legend. These errors do not affect the conclusions of the article.

DOI: 10.1523/JNEUROSCI.0976-19.2019

\section{Erratum: Battal et al., "Representation of Auditory Motion Directions and Sound Source Locations in the Human Planum Temporale"}

In the article "Representation of Auditory Motion Directions and Sound Source Locations in the Human Planum Temporale" by Ceren Battal, Mohamed Rezk, Stefania Mattioni, Jyothirmayi Vadlamudi, and Olivier Collignon, which appeared on pages 2208-2220 of the March 20, 2019 issue, the country of the second affiliation was listed incorrectly. The second affiliation should have read "Crossmodal Perception and Plasticity Laboratory, Institute for Research in Psychology, Institute of Neuroscience, Université catholique de Louvain, 1348 Louvain, Belgium”. This error does not affect the conclusions of the article. The online version has been corrected.

DOI: 10.1523/JNEUROSCI.0996-19.2019 